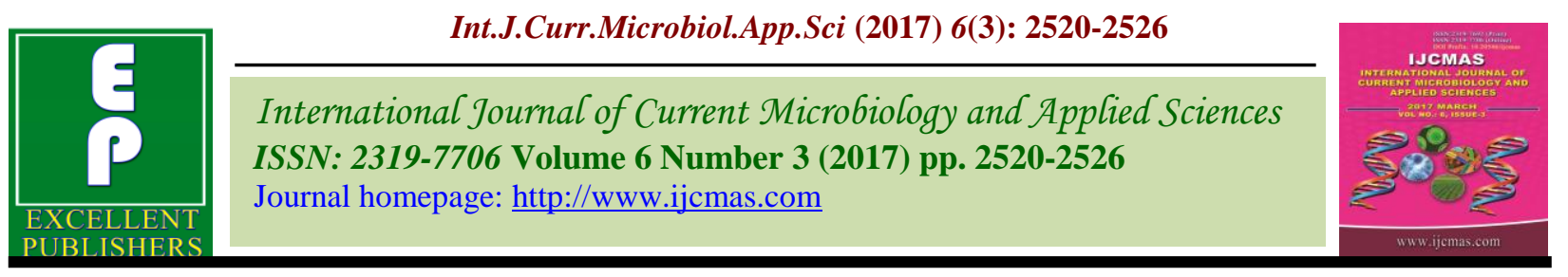

Original Research Article https://doi.org/10.20546/ijcmas.2017.603.285

\title{
Evaluation of Newer Pesticides against Leafhopper Population and its Effect on Summer Okra Yield
}

\author{
Y.T. Jadhav ${ }^{1}$, P.R. Zanwar ${ }^{2}$ and D.S. Shinde ${ }^{3}$ \\ ${ }^{1}$ Department of Agricultural Entomology, Ratnai Agriculture College, \\ Akluj -413101(M.S.), India \\ ${ }^{2}$ Department of Agricultural Entomology, Vasantrao Naik Marathwada Krishi Vidyapeeth, \\ Parbhani - 431402 (M.S.), India \\ ${ }^{3}$ Department of Agricultural Entomology, College of Agriculture, Paniv, India \\ *Corresponding author
}

Keywords

Bio-efficacy, Fenpropathrin,

Imidacloprid,

Thiamethoxam,

Diafenthiuron.

Article Info

Accepted:

20 February 2017

Available Online:

10 March 2017
The studies on bio-efficacy of leafhopper population showed quite promising results and from the pooled data at first spray it can be revealed that treatment fenpropathrin $30 \mathrm{EC}$ at $200 \mathrm{ml}$ a.i./ha was found most effective while during second spray the highest population reduction of leafhopper was obtained in imidacloprid $70 \mathrm{WG}$ at $35 \mathrm{~g}$ a.i./ha treatment. The average marketable fruit yield among different treatments ranged from 41.43 to $72.42 \mathrm{q} / \mathrm{ha}$. The highest yield was recorded by imidacloprid $70 \mathrm{WG}$ at $35 \mathrm{~g}$ a.i./ha followed by diafenthiuron $50 \mathrm{WP}$ at $600 \mathrm{~g}$ a.i./ha and thiamethoxam $25 \mathrm{WG}$ at $100 \mathrm{~g}$ a.i./ha.

\section{Introduction}

Okra (Abelmoschus esculentus (L.)Moench) originated from Africa commonly known as "Lady's finger" or "Bhendi" under Malvaceae family is a flowering plant which has multipurpose crop value producing high valued edible green pods with good nutritional. In okra cultivation and production India ranks first with an area of 532.64 thousand hectares and production of 6346.40 thousand tones alongwith productivity of $13.14 \mathrm{mt} /$ ha (Anonymous, 2013). Okra is also known as the house of pests due to its two distinct i.e vegetative and fruiting growing stages. As high as 72 species of insects have been recorded on okra. Jassid attack causes the leaves to curl upward along the tips and margins develop necrotic areas which extend over the entire leaf surface resulting in "hopper burn symptoms" while aphid and thrips reduce the vigour of the plants (Walse, 2004). Leafhoppers are one of the important pests in the early stage of the crop which desap the plants, make them weak and reduce the yield. Failure to control them in the initial stages was reported to cause an yield loss to the tune of 54.04 per cent (Chaudhary and Dadeech, 1989). 
Due to which, the present investigation were undertaken with an objective to know better management of these destructive okra sucking pests, information on effective newer insecticide, botanical and acaricide molecules are pre-requisite.

\section{Materials and Methods}

A field experiment was conducted at Department of Agricultural Entomology, Vasantrao Naik Marathwada Krishi Vidyapeeth, Parbhani to study the bioefficacy of newer pesticides against okra during summer 2013 and summer 2014.

The field design was RBD with two replications and fourteen treatments in which Mahyco Popular Bhendi No. 1 variety was sown keeping spacing of $60 \mathrm{~cm} X 60 \mathrm{~cm}$ plant to plant. Observations were made by randomly selecting 5 plants from each plot and top, middle and bottom leaves of each randomly selected plants were considered for counting number of leafhoppers. Pretreatment observations were recorded one day before the application of pesticide and post treatment observations were recorded on 1, 3, 7 and 14 days after spraying. The data were averaged and subjected to square root transformation and then statistically analyzed and the results were interpreted at five per cent level of significance by using ICAR wasp 2 software. To compare the bio-efficacy of different newer pesticides, per cent reduction in the population of leafhopper over untreated control (water spray) was calculated using Henderson and Tilton (1955) formula.

The okra fruits were ready for first picking after 45 days after sowing and near about 10 pickings were completed. The weight of fruits was recorded on individual plot basis. The recorded weight of fruits on individual plot basis was later computed to hectare basis and then subjected to statistical analysis.

\section{Results and Discussion}

The bio-efficacy data regarding leafhopper during summer 2013 and 2014 (Pooled) on okra were recorded with an objective to develop economically feasible management strategy, to reduce unwarranted pesticide load in the environment and to gain knowledge on safer pesticides during the study period.

\section{Leafhopper (Amrasca biguttula biguttula Ishida)}

\section{First spray}

In two successive cropping years, the results (Table 1) during first spray revealed significant reduction in leafhopper population at $1^{\text {st }}, 3^{\text {rd }}$ and $7^{\text {th }}$ day after application of pesticides compared to untreated check.

A day before first spray, it was observed that there was no significant difference among the treatments evaluated and the leafhopper population ranged from 6.42 to 8.16 leafhoppers/ 3 leaves. A day after first spray, among the treatments $\mathrm{T}_{6}$ (fenpropathrin 30EC) recorded the lowest leafhopper population of 1.30 leafhopper / 3 leaves whereas highest leafhopper population of 8.27 leafhopper/ 3 leaves was recorded in untreated check. The treatment fenpropathrin $30 \mathrm{EC}$ was followed by fipronil 5SC, imidacloprid 70WG and thiamethoxam 25WG which were found at par with each other. Whereas treatment $\mathrm{T}_{4}$ showed results at par with $\mathrm{T}_{3}, \mathrm{~T}_{2}, \mathrm{~T}_{1}, \mathrm{~T}_{7}, \mathrm{~T}_{12}, \mathrm{~T}_{13}, \mathrm{~T}_{5}$ and $\mathrm{T}_{8}$.

Almost similar trends were seen on third day after spraying. At 7 days after the first spray, no change in the trend was observed in which fenpropathrin 30EC showed best efficacy in controlling the leafhopper population by recording lowest leafhopper population of 2.64 leafhoppers/ 3 leaves. 
Table.1 Bio-efficacy of newer pesticides against leafhoppers, A. biguttula biguttula Ishida after first spray on okra (pooled of summer 2013 and 2014)

\begin{tabular}{|c|c|c|c|c|c|c|c|c|c|c|c|c|c|c|c|c|c|}
\hline \multirow{3}{*}{$\begin{array}{l}\text { Tr. } \\
\text { No. }\end{array}$} & \multirow{3}{*}{ Treatments } & \multirow{3}{*}{$\begin{array}{c}\text { Dose } \\
\text { (a.i./ha) }\end{array}$} & \multicolumn{15}{|c|}{ Number of leafhoppers / 3 leaves } \\
\hline & & & \multicolumn{3}{|c|}{1 DBS } & \multicolumn{3}{|c|}{1 DAS } & \multicolumn{3}{|c|}{3 DAS } & \multicolumn{3}{|c|}{7 DAS } & \multicolumn{3}{|c|}{14 DAS } \\
\hline & & & 2013 & 2014 & Pooled & 2013 & 2014 & Pooled & 2013 & 2014 & Pooled & 2013 & 2014 & Pooled & 2013 & 2014 & Pooled \\
\hline $\mathrm{T}_{1}$ & Diafenthiuron 50WP & $600 \mathrm{~g}$ & $\begin{array}{c}7.79 \\
(2.79) \\
\end{array}$ & $\begin{array}{c}6.07 \\
(2.45)\end{array}$ & $\begin{array}{c}6.93 \\
(2.63) \\
\end{array}$ & $\begin{array}{c}2.80 \\
(1.67)\end{array}$ & $\begin{array}{c}2.16 \\
(1.47)\end{array}$ & $\begin{array}{c}2.48 \\
(1.57) \\
\end{array}$ & $\begin{array}{c}2.69 \\
(1.64)\end{array}$ & $\begin{array}{c}2.10 \\
(1.44)\end{array}$ & $\begin{array}{c}2.40 \\
(1.55)\end{array}$ & $\begin{array}{c}4.23 \\
(2.05) \\
\end{array}$ & $\begin{array}{c}3.12 \\
(1.77)\end{array}$ & $\begin{array}{c}3.68 \\
(1.91)\end{array}$ & $\begin{array}{c}5.94 \\
(2.44) \\
\end{array}$ & $\begin{array}{c}6.29 \\
(2.50) \\
\end{array}$ & $\begin{array}{c}6.12 \\
(2.47)\end{array}$ \\
\hline $\mathrm{T}_{2}$ & $\begin{array}{l}\text { Thiamethoxam } \\
\text { 25WG }\end{array}$ & $100 \mathrm{~g}$ & $\begin{array}{c}7.60 \\
(2.72) \\
\end{array}$ & $\begin{array}{c}5.95 \\
(2.43) \\
\end{array}$ & $\begin{array}{c}6.78 \\
(2.60) \\
\end{array}$ & $\begin{array}{c}2.11 \\
(1.45)\end{array}$ & $\begin{array}{c}1.85 \\
(1.36) \\
\end{array}$ & $\begin{array}{c}1.98 \\
(1.41) \\
\end{array}$ & $\begin{array}{c}1.73 \\
(1.29) \\
\end{array}$ & $\begin{array}{c}1.70 \\
(1.29) \\
\end{array}$ & $\begin{array}{c}1.72 \\
(1.31)\end{array}$ & $\begin{array}{c}2.94 \\
(1.70) \\
\end{array}$ & $\begin{array}{c}2.98 \\
(1.71) \\
\end{array}$ & $\begin{array}{c}2.96 \\
(1.72) \\
\end{array}$ & $\begin{array}{c}4.93 \\
(2.22) \\
\end{array}$ & $\begin{array}{c}4.57 \\
(2.12) \\
\end{array}$ & $\begin{array}{c}4.75 \\
(2.18) \\
\end{array}$ \\
\hline $\mathrm{T}_{3}$ & Imidacloprid 70WG & $35 \mathrm{~g}$ & $\begin{array}{l}7.88 \\
(2.81) \\
\end{array}$ & $\begin{array}{c}6.15 \\
(2.48) \\
\end{array}$ & $\begin{array}{c}7.02 \\
(2.65) \\
\end{array}$ & $\begin{array}{c}2.78 \\
(1.66)\end{array}$ & $\begin{array}{c}1.31 \\
(1.09) \\
\end{array}$ & $\begin{array}{c}2.05 \\
(1.41) \\
\end{array}$ & $\begin{array}{c}2.64 \\
(1.62) \\
\end{array}$ & $\begin{array}{c}1.17 \\
(1.07)\end{array}$ & $\begin{array}{c}1.91 \\
(1.35) \\
\end{array}$ & $\begin{array}{c}3.29 \\
(1.81) \\
\end{array}$ & $\begin{array}{c}2.14 \\
(1.33) \\
\end{array}$ & $\begin{array}{c}2.72 \\
(1.64) \\
\end{array}$ & $\begin{array}{c}5.12 \\
(2.25) \\
\end{array}$ & $\begin{array}{l}4.28 \\
(2.05) \\
\end{array}$ & $\begin{array}{c}4.70 \\
(2.17) \\
\end{array}$ \\
\hline $\mathrm{T}_{4}$ & Fipronil 5SC & $1000 \mathrm{ml}$ & $\begin{array}{c}7.82 \\
(2.80) \\
\end{array}$ & $\begin{array}{c}6.07 \\
(2.46) \\
\end{array}$ & $\begin{array}{c}6.95 \\
(2.64) \\
\end{array}$ & $\begin{array}{c}2.02 \\
(1.42) \\
\end{array}$ & $\begin{array}{c}1.76 \\
(1.32) \\
\end{array}$ & $\begin{array}{c}1.89 \\
(1.37) \\
\end{array}$ & $\begin{array}{c}1.33 \\
(1.15) \\
\end{array}$ & $\begin{array}{c}2.13 \\
(1.46) \\
\end{array}$ & $\begin{array}{c}1.73 \\
(1.31) \\
\end{array}$ & $\begin{array}{c}1.90 \\
(1.36) \\
\end{array}$ & $\begin{array}{c}3.82 \\
(1.95) \\
\end{array}$ & $\begin{array}{c}2.86 \\
(1.67) \\
\end{array}$ & $\begin{array}{c}3.66 \\
(1.90) \\
\end{array}$ & $\begin{array}{c}6.48 \\
(2.54) \\
\end{array}$ & $\begin{array}{c}5.07 \\
(2.23) \\
\end{array}$ \\
\hline $\mathrm{T}_{5}$ & Buprofezin $25 \mathrm{SC}$ & $300 \mathrm{ml}$ & $\begin{array}{l}7.95 \\
(2.82) \\
\end{array}$ & $\begin{array}{c}7.04 \\
(2.65) \\
\end{array}$ & $\begin{array}{c}7.50 \\
(2.74) \\
\end{array}$ & $\begin{array}{c}2.96 \\
(1.72)\end{array}$ & $\begin{array}{c}2.54 \\
(1.59) \\
\end{array}$ & $\begin{array}{c}2.75 \\
(1.66) \\
\end{array}$ & $\begin{array}{c}2.82 \\
(1.68)\end{array}$ & $\begin{array}{c}2.42 \\
(1.53)\end{array}$ & $\begin{array}{c}2.62 \\
(1.62) \\
\end{array}$ & $\begin{array}{c}4.43 \\
(2.10) \\
\end{array}$ & $\begin{array}{c}3.71 \\
(1.92) \\
\end{array}$ & $\begin{array}{c}4.07 \\
(2.02) \\
\end{array}$ & $\begin{array}{c}6.12 \\
(2.47) \\
\end{array}$ & $\begin{array}{c}6.46 \\
(2.54) \\
\end{array}$ & $\begin{array}{c}6.29 \\
(2.51) \\
\end{array}$ \\
\hline $\mathrm{T}_{6}$ & Fenpropathrin 30EC & $200 \mathrm{ml}$ & $\begin{array}{l}7.91 \\
(2.74)\end{array}$ & $\begin{array}{c}6.11 \\
(2.40)\end{array}$ & $\begin{array}{c}7.01 \\
(2.65)\end{array}$ & $\begin{array}{c}1.81 \\
(1.34)\end{array}$ & $\begin{array}{c}0.78 \\
(0.87)\end{array}$ & $\begin{array}{c}1.30 \\
(1.11)\end{array}$ & $\begin{array}{c}2.17 \\
(1.46)\end{array}$ & $\begin{array}{c}0.62 \\
(0.79)\end{array}$ & $\begin{array}{c}1.40 \\
(1.13)\end{array}$ & $\begin{array}{c}3.64 \\
(1.90)\end{array}$ & $\begin{array}{c}1.63 \\
(1.27)\end{array}$ & $\begin{array}{c}2.64 \\
(1.59)\end{array}$ & $\begin{array}{c}5.24 \\
(2.29)\end{array}$ & $\begin{array}{c}3.62 \\
(1.86)\end{array}$ & $\begin{array}{c}4.43 \\
(2.10)\end{array}$ \\
\hline $\mathrm{T}_{7}$ & Dimethoate 30EC & $1000 \mathrm{ml}$ & $\begin{array}{c}7.62 \\
(2.76) \\
\end{array}$ & $\begin{array}{c}5.90 \\
(2.42) \\
\end{array}$ & $\begin{array}{c}6.76 \\
(2.60) \\
\end{array}$ & $\begin{array}{c}2.76 \\
(1.66) \\
\end{array}$ & $\begin{array}{c}2.25 \\
(1.49) \\
\end{array}$ & $\begin{array}{c}2.51 \\
(1.58) \\
\end{array}$ & $\begin{array}{c}2.88 \\
(1.69) \\
\end{array}$ & $\begin{array}{c}2.15 \\
(1.46) \\
\end{array}$ & $\begin{array}{c}2.52 \\
(1.58) \\
\end{array}$ & $\begin{array}{c}3.72 \\
(1.93) \\
\end{array}$ & $\begin{array}{c}3.96 \\
(1.99) \\
\end{array}$ & $\begin{array}{c}3.84 \\
(1.96)\end{array}$ & $\begin{array}{c}5.94 \\
(2.43)\end{array}$ & $\begin{array}{c}4.98 \\
(2.23)\end{array}$ & $\begin{array}{c}5.46 \\
(2.33)\end{array}$ \\
\hline $\mathrm{T}_{8}$ & Fenazaquin 10EC & $1000 \mathrm{ml}$ & $\begin{array}{c}7.99 \\
(2.83)\end{array}$ & $\begin{array}{c}6.26 \\
(2.47)\end{array}$ & $\begin{array}{c}7.13 \\
(2.66) \\
\end{array}$ & $\begin{array}{c}2.97 \\
(1.72)\end{array}$ & $\begin{array}{c}2.65 \\
(1.63) \\
\end{array}$ & $\begin{array}{c}2.81 \\
(1.68)\end{array}$ & $\begin{array}{c}4.13 \\
(2.03) \\
\end{array}$ & $\begin{array}{c}3.41 \\
(1.85)\end{array}$ & $\begin{array}{c}3.77 \\
(1.94)\end{array}$ & $\begin{array}{c}5.53 \\
(2.34) \\
\end{array}$ & $\begin{array}{c}5.53 \\
(2.35) \\
\end{array}$ & $\begin{array}{c}5.53 \\
(2.35)\end{array}$ & $\begin{array}{c}6.30 \\
(2.51)\end{array}$ & $\begin{array}{c}8.32 \\
(2.88) \\
\end{array}$ & $\begin{array}{c}7.31 \\
(2.70)\end{array}$ \\
\hline $\mathrm{T}_{9}$ & Spiromesifen 22.9SC & $400 \mathrm{ml}$ & $\begin{array}{c}7.73 \\
(2.77) \\
\end{array}$ & $\begin{array}{c}5.85 \\
(2.42)\end{array}$ & $\begin{array}{c}6.79 \\
(2.60)\end{array}$ & $\begin{array}{c}3.11 \\
(1.76)\end{array}$ & $\begin{array}{c}3.96 \\
(1.99)\end{array}$ & $\begin{array}{c}3.54 \\
(1.88)\end{array}$ & $\begin{array}{c}4.38 \\
(2.09) \\
\end{array}$ & $\begin{array}{c}3.82 \\
(1.95)\end{array}$ & $\begin{array}{c}4.10 \\
(2.02)\end{array}$ & $\begin{array}{c}5.46 \\
(2.34) \\
\end{array}$ & $\begin{array}{c}4.10 \\
(2.02)\end{array}$ & $\begin{array}{c}4.78 \\
(2.18)\end{array}$ & $\begin{array}{c}8.93 \\
(2.99) \\
\end{array}$ & $\begin{array}{c}7.27 \\
(2.69) \\
\end{array}$ & $\begin{array}{c}8.10 \\
(2.84)\end{array}$ \\
\hline $\mathrm{T}_{10}$ & Propargite 57EC & $1500 \mathrm{ml}$ & $\begin{array}{c}7.86 \\
(2.80)\end{array}$ & $\begin{array}{c}6.88 \\
(2.62)\end{array}$ & $\begin{array}{c}7.37 \\
(2.71)\end{array}$ & $\begin{array}{c}3.55 \\
(1.88)\end{array}$ & $\begin{array}{c}3.98 \\
(1.99)\end{array}$ & $\begin{array}{c}3.77 \\
(1.94)\end{array}$ & $\begin{array}{c}4.91 \\
(2.21)\end{array}$ & $\begin{array}{c}3.08 \\
(1.75)\end{array}$ & $\begin{array}{c}4.00 \\
(1.99)\end{array}$ & $\begin{array}{c}6.16 \\
(2.47)\end{array}$ & $\begin{array}{c}4.84 \\
(2.20)\end{array}$ & $\begin{array}{c}5.50 \\
(2.34)\end{array}$ & $\begin{array}{c}9.28 \\
(3.04)\end{array}$ & $\begin{array}{c}7.42 \\
(2.72)\end{array}$ & $\begin{array}{c}8.35 \\
(2.89)\end{array}$ \\
\hline $\mathrm{T}_{11}$ & Chlorfenapyr 10SC & $750 \mathrm{ml}$ & $\begin{array}{c}8.18 \\
(2.86) \\
\end{array}$ & $\begin{array}{c}8.14 \\
(2.85)\end{array}$ & $\begin{array}{c}8.16 \\
(2.86) \\
\end{array}$ & $\begin{array}{c}3.48 \\
(1.87)\end{array}$ & $\begin{array}{c}4.30 \\
(2.07)\end{array}$ & $\begin{array}{c}3.89 \\
(1.97)\end{array}$ & $\begin{array}{c}4.86 \\
(2.20) \\
\end{array}$ & $\begin{array}{c}4.12 \\
(2.03)\end{array}$ & $\begin{array}{c}4.49 \\
(2.12)\end{array}$ & $\begin{array}{c}5.33 \\
(2.31) \\
\end{array}$ & $\begin{array}{c}5.48 \\
(2.34) \\
\end{array}$ & $\begin{array}{c}5.41 \\
(2.33)\end{array}$ & $\begin{array}{c}8.75 \\
(2.96)\end{array}$ & $\begin{array}{c}8.28 \\
(2.88)\end{array}$ & $\begin{array}{c}8.52 \\
(2.92)\end{array}$ \\
\hline $\mathrm{T}_{12}$ & Dicofol 18.5EC & $1250 \mathrm{ml}$ & $\begin{array}{c}7.68 \\
(2.75) \\
\end{array}$ & $\begin{array}{c}5.16 \\
(2.27)\end{array}$ & $\begin{array}{c}6.42 \\
(2.53)\end{array}$ & $\begin{array}{c}2.94 \\
(1.71)\end{array}$ & $\begin{array}{c}2.12 \\
(1.46)\end{array}$ & $\begin{array}{c}2.53 \\
(1.59)\end{array}$ & $\begin{array}{c}2.65 \\
(1.63) \\
\end{array}$ & $\begin{array}{c}2.55 \\
(1.58) \\
\end{array}$ & $\begin{array}{c}2.60 \\
(1.61)\end{array}$ & $\begin{array}{c}4.31 \\
(2.07)\end{array}$ & $\begin{array}{c}3.92 \\
(1.98) \\
\end{array}$ & $\begin{array}{c}4.12 \\
(2.03) \\
\end{array}$ & $\begin{array}{c}8.38 \\
(2.88) \\
\end{array}$ & $\begin{array}{c}6.25 \\
(2.50) \\
\end{array}$ & $\begin{array}{c}7.32 \\
(2.70)\end{array}$ \\
\hline $\mathrm{T}_{13}$ & $\begin{array}{l}\text { Azardirachtin } \\
\text { 3000ppm }\end{array}$ & $1250 \mathrm{ml}$ & $\begin{array}{c}7.17 \\
(2.68)\end{array}$ & $\begin{array}{c}7.11 \\
(2.66)\end{array}$ & $\begin{array}{c}7.14 \\
(2.67) \\
\end{array}$ & $\begin{array}{c}2.93 \\
(1.70)\end{array}$ & $\begin{array}{c}2.34 \\
(1.53)\end{array}$ & $\begin{array}{c}2.64 \\
(1.62)\end{array}$ & $\begin{array}{c}2.71 \\
(1.65)\end{array}$ & $\begin{array}{c}2.18 \\
(1.47)\end{array}$ & $\begin{array}{c}2.45 \\
(1.56)\end{array}$ & $\begin{array}{c}3.69 \\
(1.92)\end{array}$ & $\begin{array}{l}4.30 \\
(2.07)\end{array}$ & $\begin{array}{c}4.00 \\
(2.00)\end{array}$ & $\begin{array}{c}8.40 \\
(2.90)\end{array}$ & $\begin{array}{c}6.47 \\
(2.53)\end{array}$ & $\begin{array}{c}7.44 \\
(2.72)\end{array}$ \\
\hline $\mathrm{T}_{14}$ & Untreated check & - & $\begin{array}{c}8.13 \\
(2.85)\end{array}$ & $\begin{array}{c}6.31 \\
(2.51)\end{array}$ & $\begin{array}{c}7.22 \\
(2.69) \\
\end{array}$ & $\begin{array}{c}9.39 \\
(3.06)\end{array}$ & $\begin{array}{c}7.14 \\
(2.67)\end{array}$ & $\begin{array}{c}8.27 \\
(2.87)\end{array}$ & $\begin{array}{l}10.81 \\
(3.29)\end{array}$ & $\begin{array}{l}11.72 \\
(3.41)\end{array}$ & $\begin{array}{l}11.27 \\
(3.36)\end{array}$ & $\begin{array}{l}11.38 \\
(3.37)\end{array}$ & $\begin{array}{l}14.37 \\
(3.78)\end{array}$ & $\begin{array}{l}12.88 \\
(3.58)\end{array}$ & $\begin{array}{l}16.09 \\
(4.01)\end{array}$ & $\begin{array}{l}15.92 \\
(3.99)\end{array}$ & $\begin{array}{l}16.01 \\
(4.00)\end{array}$ \\
\hline \multicolumn{3}{|c|}{ S.Em. $(\underline{+}$} & 0.25 & 0.25 & 0.11 & 0.10 & 0.14 & 0.11 & 0.14 & 0.15 & 0.13 & 0.17 & 0.21 & 0.16 & 0.16 & 0.19 & 0.15 \\
\hline \multirow{2}{*}{\multicolumn{3}{|c|}{$\mathrm{CD}$ at $5 \%$}} & NS & NS & NS & 0.32 & 0.42 & 0.34 & 0.44 & 0.45 & 0.39 & 0.54 & 0.63 & 0.48 & 0.49 & 0.59 & 0.46 \\
\hline & & & 12.92 & 13.89 & 5.88 & 8.34 & 12.15 & 9.42 & 11.04 & 12.53 & 10.42 & 11.71 & 14.29 & 10.69 & 8.48 & 10.65 & 8.06 \\
\hline
\end{tabular}

DAS - Days after spraying

Figures in the parentheses are square root transformed values 
Table.2 Bio-efficacy of newer pesticides against leafhoppers, A. biguttula biguttula Ishida after second spray on okra (pooled of summer 2013 and 2014)

\begin{tabular}{|c|c|c|c|c|c|c|c|c|c|c|c|c|c|c|c|c|c|}
\hline \multirow{3}{*}{$\begin{array}{l}\text { Tr. } \\
\text { No. }\end{array}$} & \multirow{3}{*}{ Treatments } & \multirow{3}{*}{$\begin{array}{c}\text { Dose } \\
\text { (a.i.//ha) }\end{array}$} & \multicolumn{15}{|c|}{ Number of leafhoppers/ 3 leaves } \\
\hline & & & \multicolumn{3}{|c|}{$1 \mathrm{DBS}$} & \multicolumn{3}{|c|}{1 DAS } & \multicolumn{3}{|c|}{3 DAS } & \multicolumn{3}{|c|}{7 DAS } & \multicolumn{3}{|c|}{14 DAS } \\
\hline & & & 2013 & 2014 & Pooled & 2013 & 2014 & Pooled & 2013 & 2014 & Pooled & 2013 & 2014 & Pooled & 2013 & 2014 & Pooled \\
\hline $\mathrm{T}_{1}$ & $\begin{array}{l}\text { Diafenthiuron } \\
\text { 50WP }\end{array}$ & $600 \mathrm{~g}$ & $\begin{array}{l}25.81 \\
(5.08)\end{array}$ & $\begin{array}{l}18.70 \\
(4.30)\end{array}$ & $\begin{array}{l}22.26 \\
(4.71) \\
\end{array}$ & $\begin{array}{c}8.53 \\
(2.92)\end{array}$ & $\begin{array}{c}6.18 \\
(2.48)\end{array}$ & $\begin{array}{c}7.36 \\
(2.70)\end{array}$ & $\begin{array}{c}3.71 \\
(1.92)\end{array}$ & $\begin{array}{c}5.97 \\
(2.44)\end{array}$ & $\begin{array}{c}4.84 \\
(2.19)\end{array}$ & $\begin{array}{c}5.12 \\
(2.26)\end{array}$ & $\begin{array}{c}6.97 \\
(2.64)\end{array}$ & $\begin{array}{l}6.05 \\
(2.45)\end{array}$ & $\begin{array}{l}9.16 \\
(3.03)\end{array}$ & $\begin{array}{c}8.81 \\
(2.96)\end{array}$ & $\begin{array}{c}8.99 \\
(3.00)\end{array}$ \\
\hline $\mathrm{T}_{2}$ & $\begin{array}{l}\text { Thiamethoxam } \\
\text { 25WG }\end{array}$ & $100 \mathrm{~g}$ & $\begin{array}{r}25.93 \\
(5.01) \\
\end{array}$ & $\begin{array}{l}17.19 \\
(4.15) \\
\end{array}$ & $\begin{array}{l}21.57 \\
(4.62) \\
\end{array}$ & $\begin{array}{r}3.82 \\
(1.95) \\
\end{array}$ & $\begin{array}{c}5.61 \\
(2.36) \\
\end{array}$ & $\begin{array}{c}4.72 \\
(2.16) \\
\end{array}$ & $\begin{array}{c}1.43 \\
(1.17) \\
\end{array}$ & $\begin{array}{l}4.00 \\
(2.00) \\
\end{array}$ & $\begin{array}{c}2.72 \\
(1.60) \\
\end{array}$ & $\begin{array}{c}2.36 \\
(1.50) \\
\end{array}$ & $\begin{array}{l}8.38 \\
(2.90) \\
\end{array}$ & $\begin{array}{c}5.37 \\
(2.22) \\
\end{array}$ & $\begin{array}{l}6.07 \\
(2.46) \\
\end{array}$ & $\begin{array}{l}10.19 \\
(3.19) \\
\end{array}$ & $\begin{array}{l}8.13 \\
(2.83) \\
\end{array}$ \\
\hline $\mathrm{T}_{3}$ & Imidacloprid 70WG & $35 \mathrm{~g}$ & $\begin{array}{l}25.83 \\
(5.06) \\
\end{array}$ & $\begin{array}{l}18.03 \\
(4.24) \\
\end{array}$ & $\begin{array}{l}21.93 \\
(4.67) \\
\end{array}$ & $\begin{array}{l}7.15 \\
(2.67) \\
\end{array}$ & $\begin{array}{c}3.26 \\
(1.80) \\
\end{array}$ & $\begin{array}{c}5.21 \\
(2.24) \\
\end{array}$ & $\begin{array}{c}1.96 \\
(1.40)\end{array}$ & $\begin{array}{c}2.12 \\
(1.45) \\
\end{array}$ & $\begin{array}{c}2.04 \\
(1.43) \\
\end{array}$ & $\begin{array}{c}3.02 \\
(1.73) \\
\end{array}$ & $\begin{array}{c}3.69 \\
(1.91)\end{array}$ & $\begin{array}{c}3.36 \\
(1.83) \\
\end{array}$ & $\begin{array}{c}4.81 \\
(2.19) \\
\end{array}$ & $\begin{array}{c}5.17 \\
(2.51) \\
\end{array}$ & $\begin{array}{l}4.99 \\
(2.23) \\
\end{array}$ \\
\hline $\mathrm{T}_{4}$ & Fipronil 5SC & $1000 \mathrm{ml}$ & $\begin{array}{l}26.23 \\
(5.12) \\
\end{array}$ & $\begin{array}{l}19.15 \\
(4.31) \\
\end{array}$ & $\begin{array}{l}22.69 \\
(4.76)\end{array}$ & $\begin{array}{c}6.29 \\
(2.50)\end{array}$ & $\begin{array}{c}5.57 \\
(2.36) \\
\end{array}$ & $\begin{array}{c}5.93 \\
(2.43)\end{array}$ & $\begin{array}{c}2.23 \\
(1.49)\end{array}$ & $\begin{array}{c}3.72 \\
(1.93)\end{array}$ & $\begin{array}{c}2.98 \\
(1.71)\end{array}$ & $\begin{array}{c}4.96 \\
(2.22)\end{array}$ & $\begin{array}{c}4.93 \\
(2.21)\end{array}$ & $\begin{array}{c}4.95 \\
(2.22)\end{array}$ & $\begin{array}{c}6.93 \\
(2.61)\end{array}$ & $\begin{array}{c}8.82 \\
(2.97)\end{array}$ & $\begin{array}{c}7.88 \\
(2.80) \\
\end{array}$ \\
\hline $\mathrm{T}_{5}$ & Buprofezin 25SC & $300 \mathrm{ml}$ & $\begin{array}{l}26.19 \\
(5.11) \\
\end{array}$ & $\begin{array}{l}18.18 \\
(4.26) \\
\end{array}$ & $\begin{array}{l}22.19 \\
(4.71) \\
\end{array}$ & $\begin{array}{c}9.42 \\
(3.07) \\
\end{array}$ & $\begin{array}{c}4.29 \\
(2.07) \\
\end{array}$ & $\begin{array}{c}6.86 \\
(2.57) \\
\end{array}$ & $\begin{array}{c}4.69 \\
(2.17) \\
\end{array}$ & $\begin{array}{c}3.43 \\
(1.85) \\
\end{array}$ & $\begin{array}{c}4.06 \\
(2.01) \\
\end{array}$ & $\begin{array}{c}5.79 \\
(2.40) \\
\end{array}$ & $\begin{array}{c}5.18 \\
(2.27) \\
\end{array}$ & $\begin{array}{c}5.49 \\
(2.34) \\
\end{array}$ & $\begin{array}{c}9.64 \\
(3.10)\end{array}$ & $\begin{array}{c}8.37 \\
(2.88) \\
\end{array}$ & $\begin{array}{c}9.01 \\
(3.00) \\
\end{array}$ \\
\hline $\mathrm{T}_{6}$ & Fenpropathrin 30EC & $200 \mathrm{ml}$ & $\begin{array}{l}25.90 \\
(5.08) \\
\end{array}$ & $\begin{array}{l}18.04 \\
(4.25) \\
\end{array}$ & $\begin{array}{l}21.97 \\
(4.69) \\
\end{array}$ & $\begin{array}{c}8.31 \\
(2.88) \\
\end{array}$ & $\begin{array}{c}6.50 \\
(2.54) \\
\end{array}$ & $\begin{array}{c}7.41 \\
(2.72) \\
\end{array}$ & $\begin{array}{c}6.76 \\
(2.60) \\
\end{array}$ & $\begin{array}{c}7.31 \\
(2.70) \\
\end{array}$ & $\begin{array}{l}7.04 \\
(2.65) \\
\end{array}$ & $\begin{array}{r}8.93 \\
(2.99) \\
\end{array}$ & $\begin{array}{r}7.83 \\
(2.79) \\
\end{array}$ & $\begin{array}{c}8.38 \\
(2.89) \\
\end{array}$ & $\begin{array}{c}9.06 \\
(3.01) \\
\end{array}$ & $\begin{array}{l}10.02 \\
(3.16) \\
\end{array}$ & $\begin{array}{l}9.54 \\
(3.09) \\
\end{array}$ \\
\hline $\mathrm{T}_{7}$ & Dimethoate 30EC & $1000 \mathrm{ml}$ & $\begin{array}{l}25.79 \\
(5.08)\end{array}$ & $\begin{array}{l}15.59 \\
(3.89)\end{array}$ & $\begin{array}{l}20.69 \\
(4.54)\end{array}$ & $\begin{array}{c}9.23 \\
(3.03)\end{array}$ & $\begin{array}{c}6.16 \\
(2.46)\end{array}$ & $\begin{array}{c}7.70 \\
(2.76)\end{array}$ & $\begin{array}{c}5.72 \\
(2.36)\end{array}$ & $\begin{array}{c}6.97 \\
(2.64)\end{array}$ & $\begin{array}{c}6.35 \\
(2.52)\end{array}$ & $\begin{array}{c}4.99 \\
(2.20)\end{array}$ & $\begin{array}{c}8.21 \\
(2.86)\end{array}$ & $\begin{array}{c}6.60 \\
(2.55)\end{array}$ & $\begin{array}{c}9.04 \\
(3.00)\end{array}$ & $\begin{array}{l}10.11 \\
(3.18)\end{array}$ & $\begin{array}{c}9.58 \\
(3.09) \\
\end{array}$ \\
\hline $\mathrm{T}_{8}$ & Fenazaquin 10EC & $1000 \mathrm{ml}$ & $\begin{array}{l}25.82 \\
(5.05) \\
\end{array}$ & $\begin{array}{l}18.19 \\
(4.26) \\
\end{array}$ & $\begin{array}{l}22.01 \\
(4.68) \\
\end{array}$ & $\begin{array}{c}9.31 \\
(3.05) \\
\end{array}$ & $\begin{array}{c}6.82 \\
(2.61) \\
\end{array}$ & $\begin{array}{c}8.07 \\
(2.83) \\
\end{array}$ & $\begin{array}{c}9.29 \\
(3.05) \\
\end{array}$ & $\begin{array}{c}9.29 \\
(3.04) \\
\end{array}$ & $\begin{array}{c}9.29 \\
(3.05) \\
\end{array}$ & $\begin{array}{c}9.47 \\
(3.07) \\
\end{array}$ & $\begin{array}{l}10.32 \\
(3.21) \\
\end{array}$ & $\begin{array}{c}9.90 \\
(3.15) \\
\end{array}$ & $\begin{array}{l}10.67 \\
(3.27) \\
\end{array}$ & $\begin{array}{l}12.92 \\
(3.59) \\
\end{array}$ & $\begin{array}{l}11.80 \\
(3.43) \\
\end{array}$ \\
\hline $\mathrm{T}_{9}$ & $\begin{array}{l}\text { Spiromesifen } \\
22.9 \mathrm{SC}\end{array}$ & $400 \mathrm{ml}$ & $\begin{array}{l}25.85 \\
(4.96) \\
\end{array}$ & $\begin{array}{l}19.10 \\
(4.37) \\
\end{array}$ & $\begin{array}{l}22.48 \\
(4.71) \\
\end{array}$ & $\begin{array}{l}11.07 \\
(3.31) \\
\end{array}$ & $\begin{array}{c}8.75 \\
(2.95) \\
\end{array}$ & $\begin{array}{c}9.91 \\
(3.14) \\
\end{array}$ & $\begin{array}{c}9.87 \\
(3.14) \\
\end{array}$ & $\begin{array}{l}11.17 \\
(3.33) \\
\end{array}$ & $\begin{array}{l}10.52 \\
(3.24) \\
\end{array}$ & $\begin{array}{l}10.04 \\
(3.17) \\
\end{array}$ & $\begin{array}{l}11.38 \\
(3.35) \\
\end{array}$ & $\begin{array}{l}10.71 \\
(3.27) \\
\end{array}$ & $\begin{array}{l}10.82 \\
(3.29) \\
\end{array}$ & $\begin{array}{l}13.07 \\
(3.62) \\
\end{array}$ & $\begin{array}{l}11.95 \\
(3.45) \\
\end{array}$ \\
\hline $\mathrm{T}_{10}$ & Propargite 57EC & $1500 \mathrm{ml}$ & $\begin{array}{l}25.89 \\
(5.08) \\
\end{array}$ & $\begin{array}{l}18.69 \\
(4.32) \\
\end{array}$ & $\begin{array}{l}22.29 \\
(4.72) \\
\end{array}$ & $\begin{array}{l}10.45 \\
(3.22) \\
\end{array}$ & $\begin{array}{l}7.55 \\
(2.75) \\
\end{array}$ & $\begin{array}{c}9.00 \\
(2.99) \\
\end{array}$ & $\begin{array}{l}10.71 \\
(3.27) \\
\end{array}$ & $\begin{array}{c}7.08 \\
(2.66) \\
\end{array}$ & $\begin{array}{c}8.90 \\
(2.97) \\
\end{array}$ & $\begin{array}{l}13.58 \\
(3.67) \\
\end{array}$ & $\begin{array}{c}8.93 \\
(2.99) \\
\end{array}$ & $\begin{array}{l}11.26 \\
(3.34) \\
\end{array}$ & $\begin{array}{l}14.41 \\
(3.76) \\
\end{array}$ & $\begin{array}{l}10.91 \\
(3.30) \\
\end{array}$ & $\begin{array}{l}12.66 \\
(3.55) \\
\end{array}$ \\
\hline $\mathrm{T}_{11}$ & Chlorfenapyr 10SC & $750 \mathrm{ml}$ & $\begin{array}{l}26.19 \\
(5.10) \\
\end{array}$ & $\begin{array}{l}17.20 \\
(4.14) \\
\end{array}$ & $\begin{array}{l}21.70 \\
(4.66) \\
\end{array}$ & $\begin{array}{l}10.24 \\
(3.20) \\
\end{array}$ & $\begin{array}{c}8.36 \\
(2.89) \\
\end{array}$ & $\begin{array}{c}9.30 \\
(3.05) \\
\end{array}$ & $\begin{array}{l}10.05 \\
(3.16) \\
\end{array}$ & $\begin{array}{c}6.41 \\
(2.53) \\
\end{array}$ & $\begin{array}{c}8.23 \\
(2.85) \\
\end{array}$ & $\begin{array}{l}13.37 \\
(3.64) \\
\end{array}$ & $\begin{array}{c}8.47 \\
(2.91) \\
\end{array}$ & $\begin{array}{l}10.92 \\
(3.28) \\
\end{array}$ & $\begin{array}{l}14.13 \\
(3.73) \\
\end{array}$ & $\begin{array}{l}10.19 \\
(3.19) \\
\end{array}$ & $\begin{array}{l}12.16 \\
(3.48) \\
\end{array}$ \\
\hline $\mathrm{T}_{12}$ & Dicofol 18.5EC & $1250 \mathrm{ml}$ & $\begin{array}{l}25.93 \\
(5.09) \\
\end{array}$ & $\begin{array}{l}19.64 \\
(4.43) \\
\end{array}$ & $\begin{array}{l}22.79 \\
(4.77) \\
\end{array}$ & $\begin{array}{c}9.36 \\
(3.06) \\
\end{array}$ & $\begin{array}{c}6.78 \\
(2.60) \\
\end{array}$ & $\begin{array}{c}8.07 \\
(2.83) \\
\end{array}$ & $\begin{array}{c}9.32 \\
(3.05) \\
\end{array}$ & $\begin{array}{l}10.19 \\
(3.19) \\
\end{array}$ & $\begin{array}{c}9.76 \\
(3.12) \\
\end{array}$ & $\begin{array}{c}9.59 \\
(3.09) \\
\end{array}$ & $\begin{array}{l}10.83 \\
(3.27) \\
\end{array}$ & $\begin{array}{c}10.21 \\
(3.19) \\
\end{array}$ & $\begin{array}{c}10.73 \\
(3.27) \\
\end{array}$ & $\begin{array}{l}11.82 \\
(3.44) \\
\end{array}$ & $\begin{array}{l}11.28 \\
(3.36) \\
\end{array}$ \\
\hline $\mathrm{T}_{13}$ & $\begin{array}{l}\text { Azardirachtin } \\
3000 \mathrm{ppm}\end{array}$ & $1250 \mathrm{ml}$ & $\begin{array}{l}26.09 \\
(5.11) \\
\end{array}$ & $\begin{array}{l}18.26 \\
(4.27) \\
\end{array}$ & $\begin{array}{l}22.18 \\
(4.71) \\
\end{array}$ & $\begin{array}{l}10.34 \\
(3.21)\end{array}$ & $\begin{array}{c}6.71 \\
(2.58) \\
\end{array}$ & $\begin{array}{c}8.53 \\
(2.90) \\
\end{array}$ & $\begin{array}{l}10.61 \\
(3.26) \\
\end{array}$ & $\begin{array}{c}6.17 \\
(2.48) \\
\end{array}$ & $\begin{array}{c}8.39 \\
(2.87)\end{array}$ & $\begin{array}{l}10.61 \\
(3.26) \\
\end{array}$ & $\begin{array}{r}8.65 \\
(2.94) \\
\end{array}$ & $\begin{array}{c}9.63 \\
(3.10)\end{array}$ & $\begin{array}{l}11.47 \\
(3.39) \\
\end{array}$ & $\begin{array}{l}10.26 \\
(3.20) \\
\end{array}$ & $\begin{array}{l}10.87 \\
(3.30) \\
\end{array}$ \\
\hline $\mathrm{T}_{14}$ & Untreated check & - & $\begin{array}{l}26.11 \\
(5.11) \\
\end{array}$ & $\begin{array}{l}18.54 \\
(4.29) \\
\end{array}$ & $\begin{array}{l}22.33 \\
(4.72) \\
\end{array}$ & $\begin{array}{l}28.39 \\
(5.32) \\
\end{array}$ & $\begin{array}{l}19.18 \\
(4.38) \\
\end{array}$ & $\begin{array}{l}23.79 \\
(4.85) \\
\end{array}$ & $\begin{array}{l}30.08 \\
(5.47) \\
\end{array}$ & $\begin{array}{l}19.91 \\
(4.44) \\
\end{array}$ & $\begin{array}{l}25.00 \\
(4.97) \\
\end{array}$ & $\begin{array}{l}32.12 \\
(5.66) \\
\end{array}$ & $\begin{array}{l}22.82 \\
(4.65) \\
\end{array}$ & $\begin{array}{l}27.47 \\
(5.22) \\
\end{array}$ & $\begin{array}{l}35.68 \\
(5.97) \\
\end{array}$ & $\begin{array}{l}27.17 \\
(5.21) \\
\end{array}$ & $\begin{array}{l}31.43 \\
(5.59) \\
\end{array}$ \\
\hline \multicolumn{3}{|c|}{ S.Em. $(\underline{+}$} & 0.49 & 0.34 & 0.29 & 0.21 & 0.16 & 0.18 & 0.21 & 0.17 & 0.27 & 0.23 & 0.23 & 0.29 & 0.22 & 0.21 & 0.20 \\
\hline \multirow{2}{*}{\multicolumn{3}{|c|}{$\begin{array}{l}\mathrm{CD} \text { at } 5 \% \\
\mathrm{CV} \%\end{array}$}} & NS & NS & NS & 0.63 & 0.49 & 0.54 & 0.63 & 0.52 & 0.82 & 0.71 & 0.70 & 0.90 & 0.68 & 0.64 & 0.62 \\
\hline & & & 13.58 & 11.40 & 8.71 & 9.45 & 8.56 & 8.75 & 10.94 & 9.15 & 14.23 & 11.20 & 11.02 & 14.20 & 9.60 & 8.92 & 8.76 \\
\hline
\end{tabular}

DAS - Days after spraying

Figures in the parentheses are square root transformed values 
Table.3 Effect of newer pesticide molecules on okra fruit yield of summer 2013 and 2014

\begin{tabular}{|c|l|c|c|}
\hline \multirow{2}{*}{ Tr. No. } & \multicolumn{1}{|c|}{ Treatments } & $\begin{array}{c}\text { Dose } \\
\text { (a.i./ha) }\end{array}$ & $\begin{array}{c}\text { Yield of okra } \\
\text { fruit (q/ha) }\end{array}$ \\
\cline { 4 - 4 } & & $600 \mathrm{~g}$ & 68.58 \\
\hline $\mathrm{T}_{1}$ & Diafenthiuron 50WP & $100 \mathrm{~g}$ & 67.90 \\
\hline $\mathrm{T}_{2}$ & Thiamethoxam 25WG & $35 \mathrm{~g}$ & 72.42 \\
\hline $\mathrm{T}_{3}$ & Imidacloprid 70WG & $1000 \mathrm{ml}$ & 60.49 \\
\hline $\mathrm{T}_{4}$ & Fipronil 5SC & $300 \mathrm{ml}$ & 59.92 \\
\hline $\mathrm{T}_{5}$ & Buprofezin 25SC & $200 \mathrm{ml}$ & 59.77 \\
\hline $\mathrm{T}_{6}$ & Fenpropathrin 30EC & $1000 \mathrm{ml}$ & 55.64 \\
\hline $\mathrm{T}_{7}$ & Dimethoate 30EC & $1000 \mathrm{ml}$ & 65.27 \\
\hline $\mathrm{T}_{8}$ & Fenazaquin 10EC & $400 \mathrm{ml}$ & 52.00 \\
\hline $\mathrm{T}_{9}$ & Spiromesifen 22.9SC & $1500 \mathrm{ml}$ & 53.63 \\
\hline $\mathrm{T}_{10}$ & Propargite 57EC & $750 \mathrm{ml}$ & 50.77 \\
\hline $\mathrm{T}_{11}$ & Chlorfenapyr 10SC & $1250 \mathrm{ml}$ & 64.53 \\
\hline $\mathrm{T}_{12}$ & Dicofol 18.5EC & $1250 \mathrm{ml}$ & 50.51 \\
\hline $\mathrm{T}_{13}$ & Azardirachtin 3000ppm & 41.43 \\
\hline $\mathrm{T}_{14}$ & Untreated check & - & 0.25 \\
\hline \multicolumn{3}{|c|}{ S.Em. $(+)$} & \\
\hline \multicolumn{3}{|c|}{ CD at 5\% } & \\
\hline \multicolumn{3}{|c|}{ CV \% } & 4.66 \\
\hline
\end{tabular}

However statistically it was found at par $\mathrm{T}_{3}$, $\mathrm{T}_{4}, \mathrm{~T}_{2}, \mathrm{~T}_{1}, \mathrm{~T}_{7}, \mathrm{~T}_{13}, \mathrm{~T}_{5}, \mathrm{~T}_{12}$ respectively. The treatment $\mathrm{T}_{8}$ (fenazaquin 10EC) with 5.23 leafhoppers/ 3 leaves showed the least effective results followed by $\mathrm{T}_{10}$ and $\mathrm{T}_{11}$. Leafhopper population on 14 days after the first spray ranged from 4.43 to 16.01 leafhoppers/ 3 leaves. The treatment fenpropathrin 30EC showed the lowest number of leafhopper population $(4.43$ leafhoppers/ 3 leaves) which was followed by $\mathrm{T}_{3}>\mathrm{T}_{2}>\mathrm{T}_{4}>\mathrm{T}_{7}>\mathrm{T}_{1}>\mathrm{T}_{5}$ and found statistically at par with each other. The least effective treatment recorded was $\mathrm{T}_{11}$ (chlorfenapyr 10SC) which showed maximum population incidence of 8.52 leafhoppers/ 3 leaves while in untreated check 16.01 leafhoppers/ 3 leaves were observed.
The superiority of fenpropathrin 30EC against the leafhoppers as revealed in the present study is in close agreement with Dhawan and Brar (1995) who reported that fenpropathrin $75 \mathrm{~g}$ a. i./ha was effective in controlling the sucking pests of cotton. Similar results were also obtained by Anuradha and Arjuna Rao (2005) and Shivanna et al. (2011).

\section{Second spray}

The data pooled over periods of second spray on number of leafhopper population presented in Table 2 indicated that all the treatments were significantly superior over control in reducing the leafhopper population. A day before spray showed no significant difference of leafhopper population among the evaluated 
treatments which ranged from 20.69 to 22.79 leafhoppers/ 3 leaves. After 1 day of spray, the per 3 leaves leafhopper population ranged from 4.72 to 23.79 . The most effective treatments in controlling the leafhopper was $\mathrm{T}_{2}$ (thiamethoxam 25WG) with maximum reduction of population to 4.72 leafhoppers/ 3 leaves which were found at par with $\mathrm{T}_{3}, \mathrm{~T}_{4}, \mathrm{~T}_{5}$ and $\mathrm{T}_{1}$ showing leafhopper population of 5.21, 5.93, 6.86 and 7.36 leafhoppers/ 3 leaves respectively.

The pooled data collected on 3 DAS revealed that all the treatments had significant differences with control. The least number of leafhoppers were recorded in the treatment $T_{3}$ (imidacloprid 70WG) with 2.04 leafhoppers/ 3 leaves followed by $T_{2}, T_{4}, T_{5}, T_{1}$ and $T_{7}$ which were at par with each other. Among different treatments, spiromesifen 22.9SC $\left(\mathrm{T}_{9}\right)$ recorded the highest (10.52 leafhoppers/ 3 leaves) population of leafhoppers, next to it was untreated control with 25.00 leafhoppers/ 3 leaves. Almost same trend of result was observed during seven days after spray.

By observing the pooled data regarding 14 DAS, it was evident that similar treatment as above i.e., $\mathrm{T}_{3}$ (imidacloprid 70WG) has recorded the lowest leafhoppers population of 4.99 leafhoppers/ 3 leaves followed by fipronil 5SC and thiamethoxam $25 \mathrm{WG}$ which recorded 7.88 and 8.13 leafhoppers/ 3 leaves and found at par with each other. However $\mathrm{T}_{9}$ (spiromesifen 22.9SC) and $\mathrm{T}_{11}$ (chlorfenapyr 10SC) were proved to be ineffective in controlling the leafhopper population during the consecutive years.

The present studies showed superiority of imidacloprid 70WG against the leafhoppers which are in close agreement with Gul (1998) who screened five insecticides against $A$. biguttulla biguttulla on okra and found that imidacloprid was the most effective in controlling jassids over a longer period.
Similar results were also obtained by Rathod et al. (2003) and Anitha and Nandihalli (2009).

Effect of newer pesticides molecules on fruit yield of summer okra

The ultimate output to the farmer is yield, the data pertaining on marketable fruit yield of okra for pooled results of summer 2013 and 2014 are presented in Table 3 . The results on marketable fruit yield revealed that all treatments gave significantly higher yield of okra fruits over untreated control in both the years.

Pooled data for summer 2013 and 2014 indicated that all the treatments were significantly superior over control. The average marketable fruit yield among different treatments ranged from 41.43 to $72.42 \mathrm{q} / \mathrm{ha}$. The highest yield was recorded in the treatment of $\mathrm{T}_{3}$ i.e., imidacloprid $70 \mathrm{WG}$ at $35 \mathrm{~g}$ a.i./ha (72.42 q/ha) followed by diafenthiuron 50WP at $600 \mathrm{~g}$ a.i./ha (68.58 $\mathrm{q} / \mathrm{ha}$ ) and thiamethoxam 25WG at $100 \mathrm{~g}$ a.i./ha $(67.90 \mathrm{q} / \mathrm{ha})$ which were at par with each other. The next best treatments were fenazaquin 10EC at $1000 \mathrm{ml}$ a.i./ha $(65.27$ $\mathrm{q} / \mathrm{ha}$ ) and dicofol $18.5 \mathrm{EC}$ at $1250 \mathrm{ml}$ a.i./ha $(64.53 \mathrm{q} / \mathrm{ha})$ and found at par with each other. Better yield was obtained from other treatments such as fipronil $5 \mathrm{SC}$ at $1000 \mathrm{ml}$ a.i./ha, buprofezin $25 \mathrm{SC}$ at $300 \mathrm{ml}$ a.i./ha and fenpropathrin 30EC at $200 \mathrm{ml}$ a.i./ha showing at par results with each other followed by dimethoate 30EC at $1000 \mathrm{ml}$ a.i./ha, propargite 57EC at $1500 \mathrm{ml}$ a.i./ha and spiromesifen 22.9SC at $400 \mathrm{ml}$ a.i./ha. Among different insecticidal treatments, the lower yield were recorded in the treatment of chlorfenapyr $10 \mathrm{SC}$ at $750 \mathrm{ml} / \mathrm{ha}(50.77 \mathrm{q} / \mathrm{ha})$ and azardirachtin 3000ppm at $1250 \mathrm{ml}$ a.i./ha (50.51 q/ha) both being at par with each other while untreated check plot recorded the lowest yield of $41.43 \mathrm{q} / \mathrm{ha}$. Thus increased 
level of marketable fruit yield of okra due to various treatments over untreated check is due to protection of okra crop from major sucking pests complex and also may be due to some physiological effect of pesticides on the plants leading to higher yield.

The results are in close agreement with the results obtained by Krishnaiah (1980) who reported that losses in okra due to leafhopper (A. biguttula biguttula). Failure to control okra sucking pests in the initial stages may cause yield loss to the extent of $54.04 \%$ (Chaudhary and Dadeech, 1989). The maximum yield $(508.8 \mathrm{~kg} / \mathrm{ha}, 1188.8 \mathrm{~kg} / \mathrm{ha})$ was recorded in imidacloprid ( $80 \mathrm{~g}$ a.i./ha) followed by its lower dose $40 \mathrm{~g}$ a.i./ha (455.5 $\mathrm{kg} / \mathrm{ha}, 1055.5 \mathrm{~kg} / \mathrm{ha})$ during 2004 and 2005, respectively (Raghuraman and Birah, 2011).

\section{References}

Anitha, K. R. and Nandihalli, B. S. 2008.Seasonal Incidence of Sucking Pests in Okra Ecosystem.Karnataka J. Agric. Sci., 21(1): 137-138.

Anonymous, 2013. Indian Horticultural Database 2013-14. National Horticultural Board, Ministry of Agriculture, Government of India.

Anuradha, M. and Arjuna Rao, P. 2005. Comparative Efficacy of Selected Insecticides Against Whiteflies on Brinjal. The Andhra Agric. J., 52: 173180.

Chaudhary, H.R. and Dadeech. 1989. Incidence of Insects Attacking Okra and the Available Losses Caused by them. Ann. Arid Zone., 28(3): 305-307.

Dhawan, A. K. and Brar, T. S. 1995. Effect of
Insecticides on Population Buildup of Sucking Pests during Flowering Phase of Upland Cotton Gossypium hirsutum. Pestology, 19: 8-16.

Gul, F. 1998. Evaluation of Different Insecticides and Cultivars Against Jassid in Okra. Sarhad J. Agric., 14(4): 351-354.

Henderson, C. F. And Tilton, E.W. 1955. Tests with Acaricides Against the Brown Wheat Mite.J. Econ. Entomol., 48(2):157-161.

Krishnaiah, K. 1980. Methodology for Assessing Crop Losses due to Pests of Vegetable. Assessment of Crop Losses Due to Pests and Diseases. Proc. of Workshop Held from Sept, 19-30, 1977 at U.A.S., Bangalore: 259-267.

Raghuraman M. and Birah A. 2011. Field Efficacy of Imadacloprid on Okra Sucking Pest Complex. Indian Journal of Entomology, 73(1): 76-79.

Rathod, K. S., Lavelkar, R. C., Pande, A. K., Patanage, N. R. and Sharma, O. P. 2003. Efficacy of Imidacloprid Against Sucking Pests of Cotton. Annals of Plant Protection Sciences, 11(2): 369370.

Shivanna, B.K., Naik, G., Basavaraja, M.K., Nagaraja, R., Kallevwara Swamy, C.M. and Karegowda C. 2011. Bioefficacy of New Insecticide Against Sucking Insect Pests of Transgenic Cotton. I.J.S.N., VOL. 2(1): 79-83.

Walse, M.S. 2004. Biology of Shoot and Fruit Borer and Chemical Control of Pest of Okra. M.Sc.(Agri.) Dissertion Submited to Marathwada Agricultural University Parbhani (unpublished).

\section{How to cite this article:}

Jadhav, Y.T., P.R. Zanwar and Shinde, D.S. 2017. Evalution of Newer Pesticides against Leafhopper Population and Its Effect on Summer Okra Yield. Int.J.Curr.Microbiol.App.Sci. 6(3): 2520-2526. doi: https://doi.org/10.20546/ijcmas.2017.603.285 